\title{
Could Elko Spinor Fields Induce VSR Symmetry in the DKP (Meson) Algebra?
}

\author{
R. T. Cavalcanti®*, R. da Rocha and J. M. Hoff da Silva
}

\begin{abstract}
It is shown that the meson algebra can be faced as the tensor product of Clifford algebras and, then, by constructing the DKP field by means of Elko spinors, we demonstrate that the symmetries of the so called very special relativity are inherited by the DKP field.
\end{abstract}

Mathematics Subject Classification. Primary 81T99; Secondary 81R40.

Keywords. Elko spinor fields, Clifford algebra, DKP algebra, Meson algebra, VSR symmetry.

\section{Introduction}

The very idea that dark matter fields may be understood, at least partially, within the scope of quantum field theory is quite attractive. In fact, some of the foundations of relativistic theory must be revisited in order to accomplish this program, given that the full Lorentz group irreducible representations lead to the usual quantum field theory spectrum. In the case of Elko spinors, the underlying symmetry group is the one obtained from the full Lorentz group, but without the discrete symmetries. The simple implementation of this idea, removing the parity symmetry from the formalism, leads to a fermion naturally endowed with mass dimension one. The consequences of this mirrorless world certainly deserve attention in every possible physical context, and here we attempt to investigate its consequence in the DKP (meson) algebra. We should point out that different authors use the denomination DKP algebra or meson algebra referring to the same algebraic structure. DKP is an acronym for Duffin-Kemmer-Petiau, who were the first to propose the formalism $[28,36,43]$, whereas meson algebra was the original denomination.

Work presented by Cavalcanti, R. T. in the 10th International Conference on Clifford Algebras and their Applications in Mathematical Physics.

*Corresponding author. 
Generally speaking, a DKP field is composed by usual Dirac spinors. Here, we shall modify the building blocks of the DKP field, by replacing one or more Dirac spinors by Elko(s) in the construction of the hole field. In this context, the DKP field should inherit the symmetry of the Elko field.

In order to clearly present the way how Elko spinor fields can affect the symmetries of the DKP algebra, some results concerning to Elko theory, DKP, VSR and spinor field classification need to be revised. In the next section we revisit the basics on the DKP (meson) algebra and its relation to the Clifford algebras. This will be followed by a brief discussion of Elko spinor fields and their symmetry groups, which are the very special relativity (VSR) groups. Section 4 is devoted to find linear spaces composed exclusively by Elko or Dirac spinors, which enable us to take the tensor product of those spinor spaces [19]. It is accomplished by using the Lounesto spinor field classification, introduced in the same section. Finally Elko spinor field is shown to carry signatures of VSR symmetries when we take it as constituent element of DKP theory in the Clifford algebras background. For more details we invite the reader to check Ref. [19].

\section{DKP Algebra}

The study of elementary particles by means of classical wave theory depends on the charged (either electric charge or moment dipolar charge) or uncharged aspect of the particle under consideration. A particle of mass $m$ is said to be a meson if it is described by the wave equation in Minkowski spacetime

$$
\left(\eta^{\mu \nu} \beta_{\nu} \partial_{\mu}+i m\right) \psi=0
$$

where $\beta_{\mu}$ satisfy the commutation relations $[28,36,43]$ :

$$
\beta_{\rho} \beta_{\nu} \beta_{\mu}+\beta_{\mu} \beta_{\nu} \beta_{\rho}=\eta_{\nu \mu} \beta_{\rho}+\eta_{\nu \rho} \beta_{\mu} .
$$

The DKP equation, known alternatively as meson equation, is employed to describe fields of spin 0 and 1 . There has been an increasing interest in DKP theory, after the Gribov's paper applying it to study quark confinement [32]. The basics regarding the role of the DKP theory can be checked in Refs. $[15,16,30,31,33,35,38,41,42,45]$. More details can be found in Ref. [19]. On the other hand, the Dirac equation for a spin- $1 / 2$ fermion is given by

$$
\left(\eta^{\mu \nu} \gamma_{\mu} \partial_{\mu}+i m\right) \psi=0
$$

denoting by $\left\{\gamma_{\mu}\right\}$ the set of Dirac matrices and

$$
\left\{\mathbf{1}, \gamma_{\mu}, \gamma_{\mu} \gamma_{\nu}, \gamma_{\mu} \gamma_{\nu} \gamma_{\rho}, \gamma_{0} \gamma_{1} \gamma_{2} \gamma_{3}\right\}
$$

as a basis for $\mathrm{M}(4, \mathbb{C})$, such that $\gamma_{\mu} \gamma_{\nu}+\gamma_{\nu} \gamma_{\mu}=2 \eta_{\mu \nu} \mathbf{1}$. The Clifford product is denoted by juxtaposition. The Dirac equation can be forthwith compared to the meson wave equation, via the identification $\beta_{\mu} \mapsto \gamma_{\mu}$.

From the algebraic point of view, given a quadratic space $(V, g)$ the DKP algebra $B(V, g)$ is defined as the associative algebra generated by $V$ such that

$$
x y x=g(x, y) x, \quad \text { holds } \forall x, y \in V .
$$


DKP fields given by Eqs. (2.1) and (2.2) can be expressed as the tensor product of two algebraic spinor fields, namely, elements of a minimal left ideal in the Clifford-Dirac algebra $\mathbb{C} \ell_{1,3}[19]$. In order to realize the link between DKP and Clifford algebras [34], the space $(V, g)$ can be thought as being the tangent space at a point on Minkowski spacetime. Moreover, $\mathcal{A}$ is regarded to be an associative algebra with unity $1_{\mathcal{A}}$, and $\gamma: V \rightarrow \mathcal{A}$ a linear mapping. The pair $(\mathcal{A}, \gamma)$ is a Clifford algebra $\mathcal{C} \ell(V, g)$ for $(V, g)$ when $\mathcal{A}$ is generated as an algebra by $\{\gamma(v) \mid v \in V\}$ and $\left\{a 1_{\mathcal{A}} \mid a \in \mathbb{R}\right\}$ when $\gamma(v) \gamma(u)+\gamma(u) \gamma(v)=2 g(v, u) 1_{\mathcal{A}}$, for all $v, u \in V$. For a basis $\left\{e_{\mu}\right\}$ of $V$, the element $\gamma\left(e_{\mu}\right)$ is usually denoted by $\gamma_{\mu}[40]$.

By considering the mapping $x \mapsto \frac{1}{2}(1 \otimes x+x \otimes 1)$ via an application $\delta: V \rightarrow \mathcal{C} \ell(V, g) \otimes \mathcal{C} \ell(V, g)$, where "1" stands for the identity in $\mathcal{C} \ell(V, g)$, the property

$$
\delta(u) \delta(v) \delta(u)=g(u, v) \delta(u)
$$

can be derived from $u v u=2 g(u, v) u-g(u, u) v$. By the universal property the application $\delta$ extends to an algebra monomorphism [34]

$$
\Delta: B(V, g) \rightarrow \mathcal{C} \ell(V, g) \otimes \mathcal{C} \ell(V, g)
$$

such that $\psi \in B(V, g)$ in $\frac{1}{2}(\psi \otimes 1+1 \otimes \psi)$. It is worth pointing out that, for any $v \in V$, the relation $\Delta\left(2 v^{2}-g(v, v)\right)=v \otimes v$ holds. The DKP algebra $B(V, g)$ is thus the subalgebra of $\mathcal{C} \ell(V, g) \otimes \mathcal{C} \ell(V, g)$ generated by all elements $\delta(v)[19,34]$.

The space $V$ is taken to be the Minkowski spacetime $\mathbb{R}^{1,3}$ hereupon. The associated Clifford algebra is denoted by $\mathbb{C} \ell_{1,3}$ and spinors are elements of a minimal ideal of $\mathbb{C} \ell_{1,3}$ [40]. The composition of spinors as an avatar for an element of the DKP algebra is thus an element of the algebra $\mathbb{C} \ell_{1,3} \otimes \mathbb{C} \ell_{1,3}$. DKP fields are usually expressed as the tensor product of Dirac spinor fields. Hereupon we shall depart from this assumption, and evince the unexpected role of introducing Elko spinor fields as the DKP fields building blocks.

\section{Elko and VSR}

This section is devoted to introduce basic aspects concerning Elko theory and VSR symmetries. Elko spinors are eigenspinors of the charge conjugation operator $[3,4]$. Due to its very restrict coupling possibilities with the standard model fields, except by the Higgs field, it has been proposed as a prime candidate to describe dark matter $[3,4]$.

Elko spinors can be expressed in general as (in what follows we are going to use the Weyl representation of $\gamma^{\mu}$ ) [2]

$$
\lambda\left(k^{\mu}\right)=\left(\begin{array}{c}
\sigma_{2} \phi^{*}\left(k^{\mu}\right) \\
\phi\left(k^{\mu}\right)
\end{array}\right), \quad k^{\mu} \equiv \lim _{p \rightarrow 0}(m, \mathbf{p})
$$

where $\phi\left(k^{\mu}\right)$ denotes a left-handed Weyl spinor and $p=\|\mathbf{p}\|$. Furthermore, Elko are eigenspinors of the charge conjugation operator $C$, namely, $C \lambda\left(k^{\mu}\right)=$ $\pm \lambda\left(k^{\mu}\right)$. 
The plus and minus sign regards self-conjugate and anti self-conjugate spinor fields, denoted respectively by $\lambda^{S}\left(k^{\mu}\right)$ and $\lambda^{A}\left(k^{\mu}\right)$. There are several interesting and unusual physical aspects concerning Elko theory (see $[1-7,9$, $11,13,18,20-23,25,26,37,39,44,46,47])$. The most important for our purposes here are the relations between Elko and the VSR groups. Cohen and Glashow argued that VSR, rather than special relativity, could be the fundamental symmetry of nature at the planck scale, with the standard model emerging as an effective theory [17]. Ahluwalia and Horvath, however, proposed that VSR could appear at the standard model scale, related to dark matter [5-7]. Moreover, the equation of motion for a propagating fermionic particle was studied and embedded in the framework of VSR [12].

Explicitly, given $\mathcal{J}$ and $\mathcal{K}$ the rotation and boost generators, the generators $T_{1}:=K_{x}+J_{y}$ and $T_{2}:=K_{y}-J_{x}$ can be defined. Adding $K_{z}$ to those generators yields the algebra $\mathfrak{h o m}(2)$, which is a 3 -parameter algebra associated with the group of homotheties, $\operatorname{HOM}(2)$. On the other hand, adding $J_{z}$ to $\mathfrak{h o m}(2)$ lead to a wider algebra associated to the similitude group, $\operatorname{SIM}(2)$. VSR is realized taking $\operatorname{HOM}(2)$ and $\operatorname{SIM}(2)$ as the symmetry groups of the theory. In fact, Cohen and Glashow have shown that the group $\operatorname{HOM}(2)$, which is a subgroup of $\operatorname{SIM}(2)$, is necessary and sufficient to [17]:

- Explain the results of the Michelson-Morley experiments and its more sensitive results;

- Ensure that the speed of light is the same for all observers;

- Preserve SR time dilatation and the law of addition of velocities.

Ahluwalia and Horvath have shown that Elko theory is invariant under the action of $\operatorname{HOM}(2)$ and covariant under $\operatorname{SIM}(2)$ [5]. Moreover, all VSR subgroups share the property that incorporate either P,T,CP or CT symmetries enlarging these subgroups to the full Lorentz group. An enlightening review of VSR, specially concerning Elko theory, can be found in the reference [5]. For VSR in a wider class of Lorentz violation theory see, e. g., [8].

\section{Bilinear Covariants and Lounesto Spinors Classification}

The study of Dirac and Elko spinors as constituents of the DKP algebra demands a detailed analysis of the underlying algebraic structure, requiring thus Clifford algebraic approach. The necessary tools, introduced in this section, include bilinear covariants, Lounesto classification and class preserving spaces, as can be seen below.

Let us start by regarding Minkowski spacetime $M$ and $\left\{\mathbf{e}_{\mu}\right\}\left[\left\{\theta^{\mu}\right\}\right]$ a section of the frame [dual] bundle $\mathbf{P}_{\mathrm{SO}_{1,3}^{e}}(M)$. Classical spinor fields are wellknown to carry a $(1 / 2,0) \oplus(0,1 / 2)$ representation of $\operatorname{SL}(2, \mathbb{C}) \simeq \operatorname{Spin}_{1,3}^{e}$, being sections of the vector bundle $\mathbf{P}_{\operatorname{Spin}_{1,3}^{e}}(M) \times{ }_{\rho} \mathbb{C}^{4}$. Here $\rho$ denotes the $(1 / 2,0) \oplus(0,1 / 2)$ representation of $\mathrm{SL}(2, \mathbb{C})$ as $4 \times 4$ matrices over the complex field. Hence, up to a phase factor, the multivector structure is employed to express the spinor field

$$
\psi \sim\left(\sigma+\mathbf{J}+i \mathbf{S}-i \gamma_{0123} \mathbf{K}+\gamma_{0123} \omega\right) \eta,
$$


where $\eta$ is an arbitrary spinor such that $\eta^{\dagger} \gamma_{0} \psi \neq 0[40]$, and $\sigma, \mathbf{J}, \mathbf{S}, \mathbf{K}$, and $\omega$ are the bilinear covariants provided by:

$$
\begin{aligned}
& \sigma=\psi^{\dagger} \gamma_{0} \psi, \quad \mathbf{J}=J_{\mu} \theta^{\mu}=\psi^{\dagger} \gamma_{0} \gamma_{\mu} \psi \theta^{\mu}, \quad \mathbf{S}=S_{\mu \nu} \theta^{\mu \nu}=\frac{1}{2} \psi^{\dagger} \gamma_{0} i \gamma_{\mu \nu} \psi \theta^{\mu} \wedge \theta^{\nu}, \\
& \mathbf{K}=K_{\mu} \theta^{\mu}=\psi^{\dagger} \gamma_{0} i \gamma_{0123} \gamma_{\mu} \psi \theta^{\mu}, \quad \omega=-\psi^{\dagger} \gamma_{0} \gamma_{0123} \psi .
\end{aligned}
$$

Not any combination of bilinear covariants can generate spinors, since the reconstruction from Eq. (4.1) requires the bilinear covariants must satisfy the so-called Fierz identities [40]:

$$
\mathbf{K}^{2}+\mathbf{J}^{2}=0=\mathbf{J} \times \mathbf{K}, \quad \mathbf{J}^{2}=\omega^{2}+\sigma^{2}, \quad \mathbf{K} \wedge \mathbf{J}=\left(\omega+\sigma \gamma_{0123}\right) \mathbf{S} .
$$

It turns out that the Lounesto spinor field classification provides just the

\begin{tabular}{|c|c|c|c|}
\hline$(1)$ & $\sigma \neq 0, \omega \neq 0$ & $(4)$ & $\sigma=0=\omega, \mathbf{K} \neq 0, \mathbf{S} \neq 0$ \\
\hline$(2)$ & $\sigma \neq 0, \omega=0$ & $(5)$ & $\sigma=0=\omega, \mathbf{K} \neq 0, \mathbf{S} \neq 0$ \\
\hline (3) & $\sigma=0, \omega \neq 0$ & $(6)$ & $\sigma=0=\omega, \mathbf{K} \neq 0, \mathbf{S} \neq 0$ \\
\hline
\end{tabular}
following spinor field classes [40], where in the first three classes clearly $\mathbf{J}, \mathbf{S}, \mathbf{K} \neq 0$ :

Now an important point: Lounesto spinor field classes are well known not be preserved by sum of spinor fields. For example, when two Weyl spinor fields [type-(6)] are summed it results usually in Dirac spinor fields, being among type-(1), -(2), and -(3) under Lounesto's classification. Dirac and type-(6) spinors are well known from Dirac theory and Elko is a representative of type(5) class. We note parenthetically that only in the last year a representative of the type-(4) has appeared in physics theories, in the context of ESK theories [24]. The framework that generalizes such achievements for higher dimensions is provided in Ref. [14], as well as the existing duality between different classes in a quantum gravity regime [1].

We shall analyze the space generated by tensor product of Elko linear spaces and Dirac vector spaces, however since the Lounesto spinor classification is not preserved by sum, we must find the vector space structure underlying Elko and Dirac spinor fields spaces. A natural way to accomplish this for Elko is by considering the self-conjugate and anti self-conjugate class preserving spaces, respectively. They are 4-dimensional real vector spaces, given by:

$$
\begin{aligned}
& \mathcal{E}^{S}=\operatorname{span}_{\operatorname{lin}}^{\mathbb{R}}\left\{(-i, 0,0,1)^{\top},(-1,0,0, i)^{\top},(0, i, 1,0)^{\top},(0,1, i, 0)^{\top}\right\}, \\
& \mathcal{E}^{A}=\operatorname{span}_{\operatorname{lin}}^{\mathbb{R}}\left\{(i, 0,0,1)^{\top},(1,0,0, i)^{\top},(0,-i, 1,0)^{\top},(0,-1, i, 0)^{\top}\right\} .
\end{aligned}
$$

Explicit calculations can show that merely a few combinations mixing elements of both spaces are of type-(5), but they do not correspond to Elko.

At least $\sigma$ or $\omega$ must be different of zero, for Dirac spinor fields [types(1),-(2),-(3)] under Lounesto classification, making the following 4D vector spaces to be defined:

$$
\begin{aligned}
& \mathcal{D}^{+}=\operatorname{span}_{\operatorname{lin}}^{\mathbb{R}}\left\{(1,0,1,0)^{\top},(i, 0, i, 0)^{\top},(0, i, 0,1)^{\top},(0,-1,0, i)^{\top}\right\}, \\
& \mathcal{D}^{-}=\operatorname{span}_{\operatorname{lin}}^{\mathbb{R}}\left\{(-1,0,1,0)^{\top},(-i, 0, i, 0)^{\top},(0,-i, 0,1)^{\top},(0,1,0, i)^{\top}\right\} .
\end{aligned}
$$


For a complete characterization of the spinor classes under Lounesto classification see [18], with a survey in [25]. Such classification in spaces endowed with arbitrary bilinear forms is accomplished in [1].

\section{Elko and Dirac in DKP $\rightarrow$ VSR}

Given the linear space structure of the Elko and Dirac class preserving spaces introduced in the previous section, now we are able to take the tensor product of those spaces. As we have seen in the second section, it is necessary in order to construct an DKP algebra's element using elements of the appropriate Clifford algebra. In what follow we denote $\mathcal{D}$ and $\mathcal{E}$ for the above Dirac and Elko class preserving spaces.

Operators acting on the space $\mathcal{S}_{1} \otimes \mathcal{S}_{2}$, where $\mathcal{S}_{i} \in\{\mathcal{D}, \mathcal{E}\}, i=1,2$, are defined by

$$
\mathscr{O}=\frac{1}{\sqrt{2}}\left(\mathcal{O}_{\mathcal{S}_{1}} \otimes \mathbb{I}_{\mathcal{S}_{2}}+\mathbb{I}_{\mathcal{S}_{1}} \otimes \mathcal{O}_{\mathcal{S}_{2}}\right)
$$

where $\mathcal{O}_{\mathcal{S}_{i}} \in \operatorname{End}\left(\mathcal{S}_{i}\right)$ denotes general operators in the endomorphism group of the $\mathcal{S}_{i}$ space. However, as a matter of simplification we will avoid the sub indexes $(\cdot)_{\mathcal{S}_{i}},(\cdot)_{\mathcal{D}}$, or $(\cdot)_{\mathcal{E}}$.

Thus given $\chi_{i} \in \mathcal{S}_{i}$, the action of the operator $\mathscr{O} \in \operatorname{End}\left(\mathcal{S}_{1} \otimes \mathcal{S}_{2}\right)$ is given by

$$
\mathscr{O}\left(\chi_{1} \otimes \chi_{2}\right)=\frac{1}{\sqrt{2}}\left(\mathcal{O} \chi_{1} \otimes \chi_{2}+\chi_{1} \otimes \mathcal{O} \chi_{2}\right) .
$$

In general it is straightforward to show that

$$
\mathscr{O}^{n}\left(\chi_{1} \otimes \chi_{2}\right)=2^{-\frac{n}{2}} \sum_{p=0}^{n}\left(\begin{array}{l}
n \\
p
\end{array}\right) \mathcal{O}^{n-p} \chi_{1} \otimes \mathcal{O}^{p} \chi_{2} .
$$

We are interested in the action of the discrete operators playing the role of $\mathcal{O}$. Although only the charge conjugation operator $\mathscr{C}$ may consistently act on Elko spinors [2], thereby our focus will be directed to this operator. In fact we are going to see that the charge conjugation operator acting on Elko is the source of VSR symmetries in the DKP elements constructed as above.

\subsection{Dirac $\otimes$ Elko and Elko $\otimes$ Elko}

For the charge conjugation operator $\mathscr{C} \in \operatorname{End}(\mathcal{D} \otimes \mathcal{E})$ we have [19]

$$
\mathscr{C}^{2}(\psi \otimes \lambda)= \pm \mathcal{C} \psi \otimes \lambda+\psi \otimes \lambda
$$

The sign in the above equation regards $\mathcal{C} \lambda^{S}=+\lambda^{S}$ and $\mathcal{C} \lambda^{A}=-\lambda^{A}$. When we take into account a DKP field $\lambda \otimes \psi$, the action of the operator $(\mathcal{C} \otimes \mathcal{O})$, where $\mathcal{O}$ is one of the discrete operators $(\mathcal{C}, \mathcal{P}, \mathcal{T})$, is given by

$$
\begin{aligned}
(\mathcal{C} \otimes \mathcal{O})\left(\lambda^{S} \otimes \psi\right) & =+\lambda^{S} \otimes \mathcal{O} \psi \\
(\mathcal{C} \otimes \mathcal{O})\left(\lambda^{A} \otimes \psi\right) & =-\lambda^{A} \otimes \mathcal{O} \psi .
\end{aligned}
$$


The change of sign under the action of the charge conjugation operator indicates that the Lorentz symmetry is broken in those DKP fields. Similar results appear when we act with $\mathcal{C} \otimes \mathcal{C}$ on $\lambda \otimes \lambda$

$$
\begin{aligned}
& (\mathcal{C} \otimes \mathcal{C})\left(\lambda^{S} \otimes \lambda^{S}\right)=\lambda^{S} \otimes \lambda^{S} \\
& (\mathcal{C} \otimes \mathcal{C})\left(\lambda^{A} \otimes \lambda^{S}\right)=-\lambda^{A} \otimes \lambda^{S} \\
& (\mathcal{C} \otimes \mathcal{C})\left(\lambda^{S} \otimes \lambda^{A}\right)=-\lambda^{S} \otimes \lambda^{A} \\
& (\mathcal{C} \otimes \mathcal{C})\left(\lambda^{A} \otimes \lambda^{A}\right)=\lambda^{A} \otimes \lambda^{A}
\end{aligned}
$$

Thus we see that Elko leaves a signature on the action of charge conjugation on DKP fields that neither Dirac spinors nor Majorana ones $\left(\lambda^{S}\right)$ can carry, as we can see in the above equations. In the cases above it is fairly simple to distinguish between two cases: the case when the DKP field is composed by Dirac $\otimes$ Elko, in which there is a remaining full Lorentz symmetry in the Dirac sector and the case when there is only $\operatorname{HOM}(2)$ [or $\operatorname{SIM}(2)]$ symmetries. Of course, in the former case the discrete symmetries may be applied in the Dirac sector, but it is ill defined for the full DKP field. Therefore it is more favorable to seek for VSR typical signatures from the application of the charge conjugation operator, bearing in mind the typical behavior of Elko fields under the action of $\mathcal{C}$. Thus, the results above evince that the DKP field constructed by a tensor product between Dirac and Elko or Elko and Elko spinor fields are not invariant under the full Lorentz group, but rather by the Elko's symmetry group. It shows how the Elko field induces the VSR symmetry on the DKP field.

\section{Issues on Elko Detection}

Elko is neutral spin- $1 / 2$ fermion, being an eigenspinor of the charge conjugation operator. Since Elko has mass dimension one, it can not interact with gauge fields, just with the Higgs and with the graviton as well. It makes Elko to be a prime candidate to the dark matter problem $[6,7]$, and furthermore, with inherent avoidance of experimental detection upon scales that are not cosmological. Tree level processes regard the Elko production at the LHC, stemming from a secondary level of two muons originating two Higgs bosons, and then Elkos [9,10,27]. Those processes encompass a quartic selfinteraction and a coupling with the Higgs field. By the peculiar form of the Elko propagator $[6,7]$, the typical signature of Elko at LHC demands an angular cut to be applied, breaking thus the angular isotropy. Those processes are beyond the Standard Model. Nowadays mainly two scenarios constitute the framework for the description of Elko interactions with Higgs bosons. In fact, the first scenario regards the quartic coupling scenario, for which a $10 \mathrm{MeV}$ Elko originates an adequate relic abundance that matches the measurements by WMAP [29]. The second one is the triple coupling scenario, in which the electroweak symmetry breaking mechanism generates Elko. Further details can be presented in Refs. $[9,10,27]$. 
Since Elko has such an intrincate apparatus for detection, composed bosons formed from Elko, as the ones we propose here, are even more complicated to be detected.

\section{Final Remarks}

In the usual construction, the DKP field is nothing but the combination, by means of the tensor product, of two Dirac spinors, carring a representation of Lorentz group. It turns out, however, that the (full) Lorentz group can be dismembered putting away its discrete part and the residual symmetry (without discrete transformations) can also perform well defined groups. In this vein, it is interesting to speculate whether the aforementioned symmetry can manifest itself in some composite algebra, as the one we treat in dealing with DKP fields.

Having formalised the relationship between meson and Clifford algebras we study the behavior of DKP fields composed by Elko(s). Then, taking into account the precise response of Elko spinors under the action of the charge conjugation operator, we were able to envisage a typical signature for the DKP field when composed by Elko(s) which is not shared by any other possible constituent. This is an indirect, though exhaustive, imprint of VSR symmetries into DKP algebra.

\section{Acknowledgment}

R. da Rocha is grateful to SISSA and Prof. Loriano Bonora for the hospitality, to CNPq grants 303027/2012-6 and 473326/2013-2, and is also Bolsista da CAPES Proc. $n^{o}$ 10942/13-0. J. M. Hoff da Silva thanks to CNPq (308629/2012-6; 445385/2014-6). R. T. Cavalcanti thanks to CAPES for financial support.

\section{References}

[1] Ablamowicz, R., Gonçalves, I., da Rocha, R.: Bilinear covariants and spinor fields duality in quantum clifford algebras. J. Math. Phys. 55, 103501 (2014)

[2] Ahluwalia, D. V.: On a local mass dimension one Fermi field of spin one-half and the theoretical crevice that allows it. arXiv:1305.7509

[3] Ahluwalia, D.V., Grumiller, D.: Spin half fermions with mass dimension one: Theory, phenomenology, and dark matter. JCAP 07, 012 (2005)

[4] Ahluwalia, D.V., Grumiller, D.: Dark matter: A spin one half fermion field with mass dimension one?. Phys. Rev. D 72, 067701 (2005)

[5] Ahluwalia, D.V., Horvath, S.P.: Very special relativity as relativity of dark matter: The Elko connection. JHEP 11, 078 (2010)

[6] Ahluwalia, D.V., Lee, C.-Y., Schritt, D., Watson, T.F.: Elko as self-interacting fermionic dark matter with axis of locality. Phys. Lett. B 687, 248 (2010)

[7] Ahluwalia, D.V., Lee, C.-Y., Schritt, D.: Self-interacting Elko dark matter with an axis of locality. Phys. Rev. D 83, 065017 (2011)

[8] Alfaro, J., Rivelles, V.O.: Very special relativity and lorentz violating theories. Phys. Lett. B 734, 239 (2014) 
[9] Alves, A., de Campos, F., Dias, M., Hoff da Silva, J.M.: Searching for Elko dark matter spinors at the CERN LHC. Int. J. Mod. Phys. A 30, 01, 1550006 (2015)

[10] Alves, A., Dias, M., de Campos, F.: Perspectives for an Elko phenomenology using monojets at the 14 TeV LHC. Int. J. Mod. Phys. D 23, 14, 1444005 (2014)

[11] Basak, A., Bhatt, J.R., Shankaranarayanan, S., Varma, K.V.P.: Attractor behaviour in ELKO cosmology. JCAP 04, 025 (2013)

[12] Bernardini, A.E., da Rocha, R.: Obtaining the equation of motion for a fermionic particle in a generalized Lorentz-violating system framework. Europhys. Lett. 81, 40010 (2008)

[13] Bernardini, A.E., da Rocha, R.: Dynamical dispersion relation for ELKO dark spinor fields. Phys. Lett. B 717, 238 (2012)

[14] Bonora, L., de Brito, K.P.S., da Rocha, R.: Spinor fields classification in arbitrary dimensions and new classes of spinor fields on 7Manifolds. JHEP 1502, 069 (2015)

[15] Casana, R., Fainberg, V., Lunardi, J.T., Pimentel, B.M., Teixeira, R.G.: Massless DKP fields in Riemann-Cartan space-times. Class. Quantum Grav 20, 2457 (2003)

[16] Castro, L.B., de Castro, A.S.: Corroborating the equivalence between the Duffin-Kemmer-Petiau and the Klein-Gordon and Proca equations. Phys. Rev. A 90, 022101 (2014)

[17] Cohen, A.G., Glashow, S.L.: Very special relativity. Phys. Rev. Lett. 97, 021601 (2006)

[18] Cavalcanti, R.T.: Classification of singular spinor fields and other mass dimension one fermions. Int. J. Mod. Phys. D 23, 1444002 (2014)

[19] Cavalcanti, R.T., da Silva, J.M.H., da Rocha, R.: VSR symmetries in the DKP algebra: the interplay between Dirac and Elko spinor fields. Eur. Phys. J. Plus 129, 246 (2014)

[20] da Rocha, R., da Silva, J.M.H.: From dirac spinor fields to ELKO. J. Math. Phys. 48, 123517 (2007)

[21] da Rocha, R., da Silva, J.M.H.: Hawking radiation from Elko particles tunnelling across black strings horizon. Europhys. Lett. 107, 50001 (2014)

[22] da Rocha, R., Pereira, J.G.: The Quadratic spinor Lagrangian, axial torsion current, and generalizations. Int. J. Mod. Phys. D 16, 1653 (2007)

[23] da Rocha, R., Bernardini, A.E., da Silva, J.M.H.: Exotic dark spinor fields. JHEP 04, 110 (2011)

[24] da Rocha, R., Fabbri, L., da Silva, J.M.H., Cavalcanti, R.T., SilvaNeto, J.A.: Flag-Dipole spinor fields in ESK gravities. J. Math. Phys. 54, 102505 (2013)

[25] da Silva, J.M.H., da Rocha, R.: Unfolding physics from the algebraic classification of spinor fields. Phys. Lett. B 718, 1519 (2013)

[26] da Silva, J.M.H., Pereira, S.H.: Exact solutions to Elko spinors in spatially flat Friedmann-Robertson-Walker spacetimes. JCAP 03, 009 (2014)

[27] Dias, M., de Campos, F., da Silva, J.M.H.: Exploring Elko typical signature. Phys. Lett. B 706, 352 (2012)

[28] Duffin, R.J.: On the characteristic matrices of covariant systems. Phys. Rev. 54, 1114 (1938) 
[29] Dulaney, T.R., Perez, P.F., Wise, M.B.: Dark matter, baryon asymmetry, and spontaneous B and L breaking. Phys. Rev. D 83, 023520 (2011)

[30] Fainberg, V.Y., Pimentel, B.M.: On equivalence of Duffin-Kemmer-Petiau and Klein-Gordon equations. Theor. Math. Phys. 124, 1234 (2000)

[31] Fischbach, E., Louck, J.D., Nieto, M.M., Scott, C.K.: The lie algebra so(n) and the duffin-kemmer-petiau ring. J. Math. Phys. 15, 60 (1974)

[32] Gribov, V.: QCD at large and short distances (annotated version). Eur. Phys. J. 71, C10 (1999)

[33] Harish-Chandra: The correspondence between the particle and the wave aspects of the meson and the photon. Proc. R. Soc. Lond. A 186, 502 (1946)

[34] Jacobson, N.: Structure and representations of Jordan algebras. Amer. Math. Soc. 39 (1968)

[35] Jena, P.K., Naik, P.C., Pradhan, T.: Photon as the zero mass limit of Dkp field. J. Phys. A 13, 2975 (1980)

[36] Kemmer, N.: The particle aspect of meson theory. Proc. R. Soc. Lond. A 173, 91 (1939)

[37] Kouwn, S., Lee, J., Lee, T.H., Oh, P.: ELKO spinor model with torsion and cosmology. Mod. Phys. Lett. A 28, 1350121 (2013)

[38] Krajcik, R.A., Nieto, M.M.: Historical development of the bhabha first order relativistic wave equations for arbitrary spin. Am. J. Phys. 45, 818 (1977)

[39] Liu, Y.-X., Zhou, X.-N., Yang, K., Chen, F.-W.: Localization of 5D Elko spinors on minkowski branes. Phys. Rev. D 86, 064012 (2012)

[40] Lounesto, P.: Clifford Algebras and Spinors. Cambridge University Press, Cambridge (2002)

[41] Nedjadi, Y., Barrett, R.C.: Solution of the central field problem for a DuffinKemmer-Petiau vector boson. J. Math. Phys. 35, 4517 (1994)

[42] Nedjadi, Y., Barrett, R.C.: The Duffin-Kemmer-Petiau oscillator. J. Phys. A 27, 4301 (1994)

[43] Petiau, G.: University of Paris thesis, Académie Royale De Belgique. Classe Des Sciences. Mémoires. Collection 16, 1114 (1936)

[44] Sperança, L.D.: An identification of the dirac operator with the parity operator. Int. J. Mod. Phys. D 23, 1444003 (2014)

[45] Umezawa, H.: Quantum Field Theory. North-Holland Publishing Company, Amsterdam (1956)

[46] Vignolo, S., Fabbri, L., Cianci, R.: Dirac spinors in Bianchi-I f(R)-cosmology with torsion. J. Math. Phys. 52, 112502 (2011)

[47] Wunderle, K.E., Dick, R.: Transformation properties and symmetry behaviour of ELKO spinors. Can. J. Phys. 87, 909 (2009)

R. T. Cavalcanti

Centro de Ciências Naturais e Humanas

UFABC

Santo André

$\mathrm{SP}$

09210-580

Brazil

e-mail: rogerio.cavalcanti@ufabc.edu.br 
R. da Rocha

Centro de Matemática, Computação e Cognição

UFABC

Santo André

SP

09210-580

Brazil

e-mail: roldao.rocha@ufabc.edu.br

J. M. Hoff da Silva

Departamento de Física e Quimica

UNESP

Av. Dr. Ariberto Pereira da Cunha, 333

Guaratinguetá

SP

12516-410

Brazil

e-mail: hoff@feg.unesp.br

Received: January 29, 2015.

Accepted: May 09, 2015. 